\title{
RELACIÓN ENTRE EL NIVEL DEL LAGO Y LA PRECIPITACIÓN SOBRE LOS DESEMBARQUES DEL PEJERREY Odontesthes bonariensis (VALENCIENNES, 1835) EN EL SECTOR PERUANO DEL LAGO TITICACA ENTRE 1981 Y 2010
}

\section{RELATIONSHIP BETWEEN LAKE WATER LEVEL AND RAINFALL ON THE LANDINGS OF ARGENTINIAN SILVERSIDE Odontesthes bonariensis (VALENCIENNES, 1835) IN THE PERUVIAN SECTOR OF THE TITICACA LAKE FROM 1981 TO 2010}

\author{
René Chura-Cruz ${ }^{1,4}$, Luís A. Cubillos S. ${ }^{2}$ Jorge Tam M. ${ }^{3}$, Marceliano Segura Z. ${ }^{4}$ y Carmen Villanueva Q. ${ }^{4}$
}

\section{Resumen}

El lago Titicaca es el segundo lago más grande y navegable en Sudamérica, con una superficie $\sim 8.400 \mathrm{~km}^{2}$ y localizado a una altitud de 3810 m.s.n.m. El objetivo del trabajo fue determinar si se encuentran relación entre la variabilidad del nivel del lago y la precipitación sobre los desembarques de la pesquería del pejerrey Odontesthes bonariensis en el sector peruano del lago entre 1981 y 2010, considerando la siguiente hipótesis: los cambios temporales del nivel de agua del Lago Titicaca y la precipitación tienen relación con la abundancia y disponibilidad del pejerrey, con consecuencias en los desembarques en el sector peruano del lago. Se asume que los desembarques son un índice de la abundancia. Las estadísticas de desembarques anuales de pejerrey cubrieron el periodo 1981 - 2010 y mensuales desde enero de 1990 a diciembre del 2010. Para las series anuales se eliminó la tendencia utilizando un modelo lineal, mientras las series mensuales fueron descompuestas. La relación entre las variables se basó en los residuales, utilizando técnicas de regresión, autocorrelación y correlación cruzada. Se determinó que los desembarques del pejerrey presentaron una relación significativa con el nivel del lago después de tres años. Los residuales mensuales de desembarques mostraron una correlación significativa con la precipitación con 18 meses de desfase. De la relación desfasada de tres años en el periodo 19812004, se encontró una relación significativa, y que esta relación se deteriora para el periodo 2005 2010, por lo que se establece que el cambio en la relación sería consecuencia en la disminución de la abundancia y/o productividad del pejerrey. La ampliación de la zona litoral por procesos de crecida del nivel del lago favorecería el éxito del reclutamiento del pejerrey principalmente por dos mecanismos: por la disponibilidad de alimento natural y refugio frente a depredadores.

Palabras clave: Pejerrey, Lago Titicaca, nivel de agua, precipitación, regresión, correlación.

\begin{abstract}
The Lake Titicaca is the second bigger and navigable lake in South, with a surface of 8.400 $\mathrm{km}^{2}$ and located at an altitude of $3810 \mathrm{~m}$.a.s.1. The objective of this work was to determine the relationship between fluctuations in the lake level and rainfall on the landings of the argentine silverside Odontesthes bonariensis in the Peruvian sector of the lake, between 1981 and 2010, considering the following hypothesis: the temporal changes of the water level of Lake Titicaca and rainfall are related to the abundance and availability of argentine silverside, with consequences in landings in the Peruvian sector of the lake.

It is assumed that landings are an index of abundance. The annual catch records of argentine silverside covered the period 1981 - 2010 and monthly catch data covered from January 1990 to December 2010. To the annual time series, the linear trend was removed and residuals were calculated; while the monthly time series were decomposed. All the relationships between variables were based on residuals by using regression techniques, autocorrelation and crosscorrelation functions. It was determined that the landings of argentine silverside presented a significant relationship with the lake level after three years. . The monthly landings residuals were correlated with precipitation residuals at 18 months lag. From the lagged relationship at three years lagged in the period 1981-2004, a significant linear relationship was found, but this relationship was deteriorated when the most recent period $(2005$ - 2010) was included. It is established that this change in the relationship would be due to the reduced abundance and/or productivity of argentine silverside. The expansion of the coastal zone by processes associated with lake level rise
\end{abstract}


would be favoring the recruitment success of argentine silverside through two main mechanisms: natural food availability and refuge against predators.

Key words: argentine silverside, Lake Titicaca, level, precipitation, regression, correlation.

\section{Introducción.}

El desembarque de los stocks de peces e invertebrados marinos fluctúan en patrones temporales complejos, con una variabilidad en escala de tiempo estacional, interanual, interdecadal y de más largo plazo (Pyper \& Peterman, 1998; Alheit \& Bakun, 2010). Las variaciones interanuales en las condiciones oceanográficas, limnográficas y atmosféricas son capaces de producir importantes fluctuaciones en el reclutamiento de peces y, en consecuencia, en la biomasa de las poblaciones (Quiñones \& Montes, 2001; Alheit \& Bakun, 2010).

En aguas continentales, el efecto del ambiente sobre las poblaciones de peces ha sido poco estudiado. Las fluctuaciones del nivel del agua de los lagos y ríos, se caracterizan por su frecuencia y duración, y constituyen el forzante principal que controla el funcionamiento de estos ecosistemas, con énfasis en los procesos de interface de la zona litoral de los recursos acuáticos y terrestres (Leira \& Cantonati, 2008).

El lago Titicaca es el segundo lago más grande y navegable de Sudamérica $\left(15^{\circ} 13^{\prime}\right.$ $16^{\circ} 36^{\prime} \mathrm{S}, 68^{\circ} 34^{\prime}-70^{\circ} 02^{\prime}$ W) con una superficie de aproximadamente $8400 \mathrm{~km}^{2}$, y un volumen de $930 \mathrm{~km}^{3}$ y localizado a una altitud de $3810 \mathrm{~m}$ sobre el nivel del mar (Hahmenberger, 2003) (Figura 1). Comprende dos cuencas, una conocida como el Lago Mayor (6 500 $\mathrm{km}^{2}$ ), donde se encuentra la máxima profundidad (284 m); y la segunda, como el Lago Pequeño $\left(1400 \mathrm{~km}^{2}\right)$ que cubre $16 \%$ de la superficie total, con una profundidad media entre 20 y $30 \mathrm{~m}$, y está conectado al Lago Mayor a través del estrecho de Tiquina (Figura 1) Una tercera región está representada por la Bahía de Puno, con $500 \mathrm{~km}^{2}$ (Paredes \& Gonfiantini, 1999).

El rol de la precipitación en el Lago Titicaca es fundamental es favorecida por la topografía y las variaciones en la reflectividad de la superficie del lago (Garreaud \& Aceituno, 2001). La precipitación provee el 55\% del agua hacia el lago (Paredes \& Gonfiantini, 1999). Las fluctuaciones interanuales de la precipitación sobre el Altiplano, se extienden a lo largo de los Andes Central y Ecuatorial, y las anomalías de viento de la zona bajan sobre el margen occidental de Sudamérica tropical y Pacífico Oriental, donde muestra una relación con la zona Este/húmedo y Oeste/seco.
El pejerrey, Odonthestes bonariensis (Valenciennes, 1835), constituye la primera especie de importancia pesquera en el Lago Titicaca. El pejerrey es una especie que habita los territorios de Argentina, Uruguay y el sur de Brazil. En la Argentina ocupa las lagunas y ríos de la región de la pampasia como así es frecuente en los ríos de Paraná, Uruguay y de la Plata.

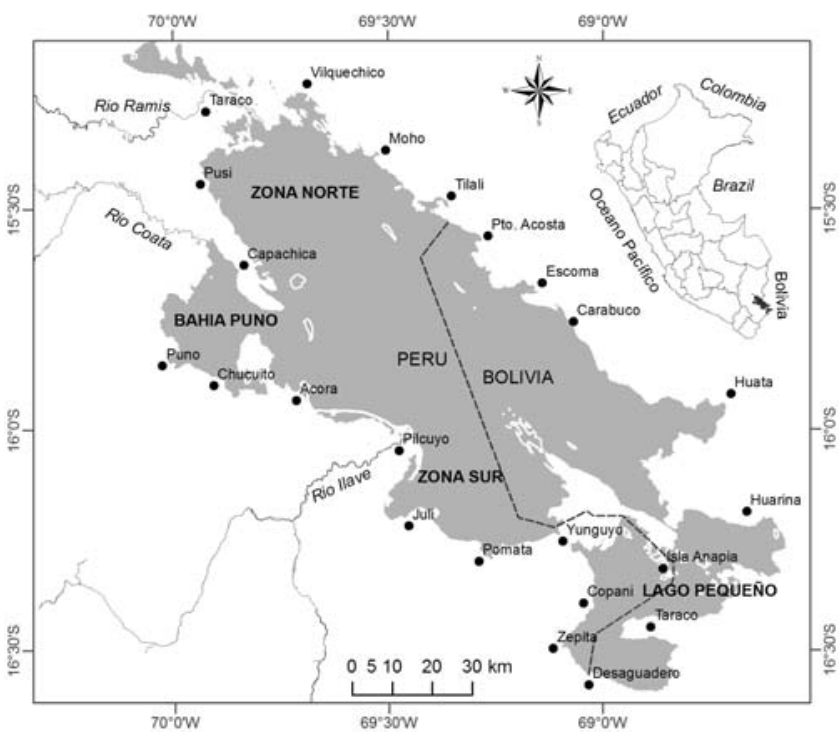

$70^{\circ} 0 \mathrm{w}$

$\mathrm{Su}$ introducción al Lago Titicaca tuvo origen en el Lago Poopo - introducido en 1946 por un Club de Pesca Boliviano - y migró al lago a través del río Desaguadero en 1955 (Loubens \& Osorio, 1991).

El pejerrey crece a un tamaño asintótico de $47 \mathrm{~cm}$ de longitud total (LT), y con una tasa de crecimiento moderada $\left(\mathrm{K}=0.23 \mathrm{año}^{-1}\right)$ (IMARPE, 2008; Flores, 2010), lo que permite inferir que el reclutamiento al arte de pesca podría ocurrir entre 1 y 3 años de edad, y a tamaños de entre 15 y $26 \mathrm{~cm} \mathrm{LT}$, respectivamente. Es una especie de hábitos pelágicos en su fase adulta, mientras que los juveniles se distribuyen preferentemente en la zona litoral $(<15 \mathrm{~m}$ de profundidad), en el cauce de los ríos Ramis e Ilave en Perú, y Suches en Bolivia, en asociación con la biota de macrófitas (totora, chara y Potamogeton sp.) (Calsina et al., 2001; Deguen et al., 2002; IMARPE, 2009). En etapa adulta se alimentan de peces como el ispi y de crustáceos como anfípodos y copépodos; mientras que en etapa juvenil consume mayoritariamente cladóceros (Vaux et al., 1988; IMARPE, 2009).

La época reproductiva principal del pejerrey en el Lago Titicaca se extiende desde julio a septiembre, y 
otra actividad secundaria desde enero a marzo (IMARPE, 2009). Cada hembra adulta (4 a 5 años) puede poner entre 35000 y 40000 huevos por desove, y los huevos tienen una corona de filamentos pegajosos, de modo que se aglutinan formando un racimo que se hunde y adhiere a la vegetación acuática. La eclosión de los huevos ocurre entre 20 y 30 días, y los alevines buscan refugio inmediatamente en la vegetación acuática presente (Yucra, 1993; Grossman, 2001).

El objetivo de este trabajo fue determinar si se encuentran relación entre la variabilidad del nivel de agua del lago Titicaca y la precipitación sobre los desembarques de la pesquería de pejerrey Odontesthes bonariensis en el sector peruano del lago entre 1981 y 2010, considerando la siguiente hipótesis: los cambios temporales del nivel de agua del Lago Titicaca y la precipitación tienen relación con la abundancia y disponibilidad del pejerrey, con consecuencias en los desembarques en el sector peruano del lago.

\section{Materiales y métodos.}

Series de tiempo históricos.

Las estadísticas de desembarques anuales de pejerrey cubren el período 1981 - 2010, y mensuales desde enero de 1990 a diciembre de 2010. Estos datos se obtuvieron de los Anuarios Estadísticos de la Dirección Regional de Producción de Puno (DIREPRO PUNO) y del Instituto del Mar del Perú (IMARPE).

Los datos del nivel de agua del Lago Titicaca y precipitación se obtuvieron de los registros del Servicio Nacional de Meteorología e Hidrología (SENAMHI). Se utilizó datos mensuales del nivel de agua del lago y de la precipitación con el objeto de conformar una serie de tiempo que cubra el periodo de registros de desembarque de pejerrey. En relación con la especie objetivo de estudio, el pejerrey (Odontesthes bonariensis) representó para el período 1981 y 2010 un $43.4 \%$ del total capturado, equivalente a 46178 t (Tabla 1), siendo la especie más capturada de un total de once especies del Lago Titicaca. De los reportes del IMARPE entre 2007 y 2010, el pejerrey fue capturado, principalmente con redes cortina $(90 \%$, $15-40 \mathrm{~cm} \mathrm{LT})$, seguido del espinel $(8 \%, 20-30 \mathrm{~cm}$ LT) y chinchorro de playa (4 - $12 \mathrm{~cm} \mathrm{LT})$.

Tabla 1. Especies de mayor desembarque en la pesquería artesanal del Lago Titicaca entre 1981 y 2010

\begin{tabular}{lrr}
\hline \multicolumn{1}{c}{ Nombre Científico } & $\begin{array}{c}\text { Desembarque } \\
\text { total (t) }\end{array}$ & $\begin{array}{c}\text { Desembarque } \\
\text { total (\%) }\end{array}$ \\
\hline Pejerrey Odontesthes bonariensis & 46178 & 43.4 \\
Ispi Orestias ispi & 12460 & 11.7 \\
Mauri Trichomycterus dispar & 1863 & 1.8 \\
Trucha Oncorhynchus mykiss & 1548 & 1.5 \\
Boga Orestias pentlandii & 926 & 0.9 \\
Otros 6 spp. (Carachis) & 43358 & 40.8 \\
Total & 106333 & 100.0 \\
\hline
\end{tabular}

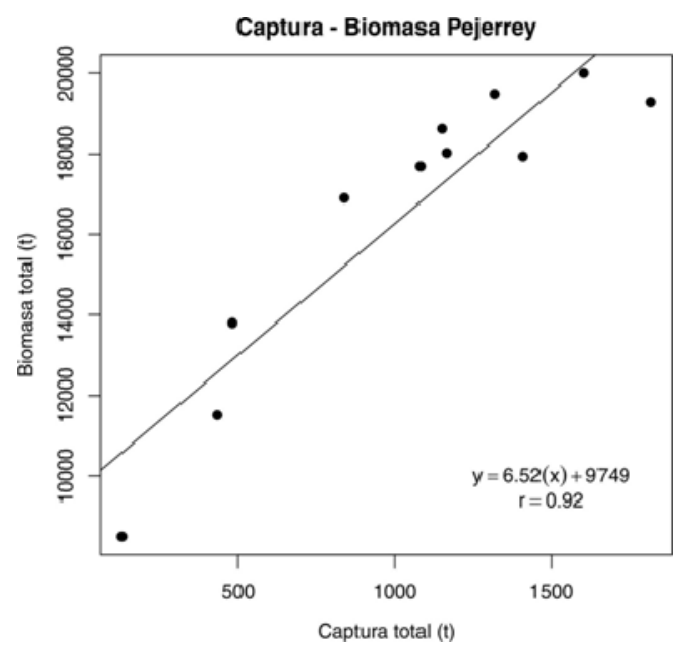

Figura 2. Relación entre los desembarques y biomasas totales anuales de pejerrey Odontesthes bonariensis en el Lago Titicaca.

Los datos de desembarque anuales están orientados a establecer las relaciones interanuales entre el nivel de agua del lago y la abundancia, mientras que las series de tiempo mensuales permitirán evaluar relaciones desfasadas que den cuenta de cambios en la disponibilidad estacional. Los desembarques como índice de abundancia.

Los datos de desembarque como índice de abundancia pueden ser cuestionados en escalas de tiempo cortas (meses), ya que pueden estar determinadas por múltiples factores tales como la disponibilidad, el esfuerzo de pesca, precios, entre otros (Fréon et al., 2008). En este estudio se usó los registros de desembarque ante la ausencia de una estadística detallada del esfuerzo de pesca. Se asume que los desembarques anuales están relacionados con la abundancia, mientras que los datos mensuales de desembarque estarían principalmente relacionados con la disponibilidad a la pesca. Para validar el uso de los desembarques como índice de abundancia se estableció una relación entre los desembarques anuales y estimaciones de biomasa realizadas con el método hidroacústico. Los datos de biomasa acústica fueron obtenidos de los informes técnicos sobre las evaluaciones realizadas por el Instituto del Mar del Perú. La relación fue lineal y significativa $(\mathrm{r}=$ 0.92 , Figura 2), demostrándose que los datos de desembarque anual podrían dar cuenta de cambios en abundancia.

Áreas de inundación producto de las fluctuaciones en el nivel de agua del lago.

Con el objeto de determinar las áreas de inundación, entre 1981 y 2010, se digitalizó imágenes satelitales LANDSAT 5-6 del National Institute for Space Research of Brazil (http://www.dgi.inpe.br/CDSR/). Las imágenes 
satelitales fueron visualizadas y georeferenciadas con el programa ArcGIS versión 9.3, y luego digitalizadas considerando el borde costero del lago en los diferentes periodos. Con los mapas digitalizados se obtuvo el área total del Lago Titicaca, las cuales sirvieron para determinar el área de inundación. El área de inundación se calculó como la diferencia del área total del año y el área del lago a la cota histórica de 85 años que corresponden a 3809.9 metros sobre el nivel del mar (msnm) equivalente a $8400 \mathrm{~km}^{2}$. Posteriormente, se correlacionó las áreas de inundación con los cambios en el nivel del lago, y la abundancia del pejerrey.

Procedimiento estadístico.

Para establecer la relación entre los desembarques anuales de pejerrey y las fluctuaciones en el nivel de agua del lago se eliminó la tendencia utilizando un modelo lineal y se calculó los residuales. Posteriormente se calcularon las correlaciones cruzadas con la finalidad de determinar el grado de desfase entre las series de tiempo. Una vez identificado el desfase temporal, se analizó el grado de asociación. El nivel de significancia (valor p) fue corregido para tomar en cuenta la autocorrelación de las series y la función de autocorrelación (FAC) permitió detectar el grado de dependencia de las observaciones de las series de tiempo, y determinar el número de observaciones independientes disponibles (Pyper \& Peterman, 1998).

Finalmente, con el objeto de analizar la relación entre las series de tiempo, se utilizaron las series de tiempo mensuales de nivel del lago, precipitación y desembarques de pejerrey. Para ello, se asumió que las series siguen un modelo aditivo:

$$
Y_{i}=T_{i}+S_{i}+R_{i} \text {, donde } i=1, \ldots, N
$$

Donde: Yi es la variable de interés en el tiempo $i$, $\mathrm{T}$ es la tendencia, $\mathrm{S}$ es el componente estacional $\mathrm{S}_{\mathrm{i}}=$ $\mathrm{S}_{(\mathrm{i}+\mathrm{T})}$ donde $\mathrm{T}$ se usa para definir el horizonte, $\mathrm{y}$ Ri son los residuales no explicados por la tendencia ni el componente estacional. Para la descomposición se usó el procedimiento de Cleveland et al. (1990) implementado en el paquete 'stats' de R 2.13 (R Development Core Team, 2010), que se basa en suavizadores loess, y considera un algoritmo iterativo que suaviza y mejora progresivamente las estimaciones de la tendencia y del componente estacional, siendo resistente a observaciones extremas. Se eliminó la tendencia y la estacionalidad de los datos utilizando la función STL (Seasonal, Trend and irregular components using LOESS) de la biblioteca STATS disponible para el programa R (R Development Core Team 2010).

Con los residuales se calculó la función de autocorrelación (FAC) para determinar el grado de dependencia hasta un retraso máximo de 12 meses. La autocorrelación se evalúo considerando como hipótesis nula que si la secuencia se distribuye normal, independiente e idénticamente distribuida (IID), cerca del $95 \%$ de los datos deberían caer entre los limites de confianza, si más de 1 valor cae fuera de los limites se rechaza la hipótesis de que los datos son IID (Brockwell \& Davis, 2002). Posteriormente se utilizó una función de correlación cruzada (FCC) para detectar la dependencia entre los residuales de las series de tiempo, considerando los residuales de desembarque como dependientes y los residuales del nivel del lago, así como los de precipitación, como independientes. El objetivo de FCC fue establecer relaciones desfasadas en la respuesta de la variable dependiente en función de cambios en las variables independientes.

\section{Resultados.}

Fluctuaciones interanuales y relación entre series anuales.

El nivel del Lago Titicaca exhibió importantes fluctuaciones en el periodo 1981-2010 (Figura 3a), observándose un nivel máximo en 1986 (3 811.83 msnm) y otro secundario en 2004 (3 $810.51 \mathrm{msnm}$ ); y registros mínimos, en 1996 (3 $808.51 \mathrm{msnm}$ ) y 2010 (3 $808.92 \mathrm{msnm}$ ). La amplitud promedio anual fue de $0.9 \mathrm{~m}$, con una amplitud máxima de $1.6 \mathrm{~m}$ (2001) y mínimos de $0.5 \mathrm{~m}$ (1993). En los registros de precipitación anual para el período 1981-2010 (Figura 3b), se estableció que los años lluviosos fueron 1984 (1 $263.1 \mathrm{~mm}$ ) y 2001 (1 $019.8 \mathrm{~mm})$, y los periodos menos lluviosos fueron 1983 (428.8 mm) y 1992 (556.9 mm).

Los volúmenes de desembarque del pejerrey, entre 1981 y 2010 , muestran una fase de crecimiento desde 1981 (202 t) a 1990 (4 350 t), para posteriormente exhibir un descenso hasta el 2004 (Figura 3c), y posteriormente una caída importante en el 2005. En el 2007, se observó una recuperación (1 191 t), pero posteriormente el desembarque de pejerrey alcanza sus niveles más bajo en 2009 (68 t) y se mantiene baja en el 2010.

Se observó que los cambios en el nivel del lago están asociados significativamente con la precipitación hasta después de dos años, lo que implica que en la escala anual los años lluviosos determinan el nivel del lago hasta dos años después en forma significativa (Figura 4a). A su vez, los desembarques de pejerrey presentaron una relación significativa con los cambios en el nivel de agua del lago después de tres años (Figura 4b).

En la escala anual, el nivel del lago Titicaca presentó una estructura de autocorrelación significativa y positiva al primer $(\mathrm{r}=0.83, \mathrm{P}<0.05)$ y segundo año de retraso $(\mathrm{r}=0.59, \mathrm{P}<0.05)$, seguido con una correlación inversa después de ocho $(\mathrm{r}=-0.45$, $\mathrm{P}<0.05)$ y once años de retraso $(\mathrm{r}=-0.45, \mathrm{p}<0.05)$. Los desembarques de pejerrey exhiben la misma 

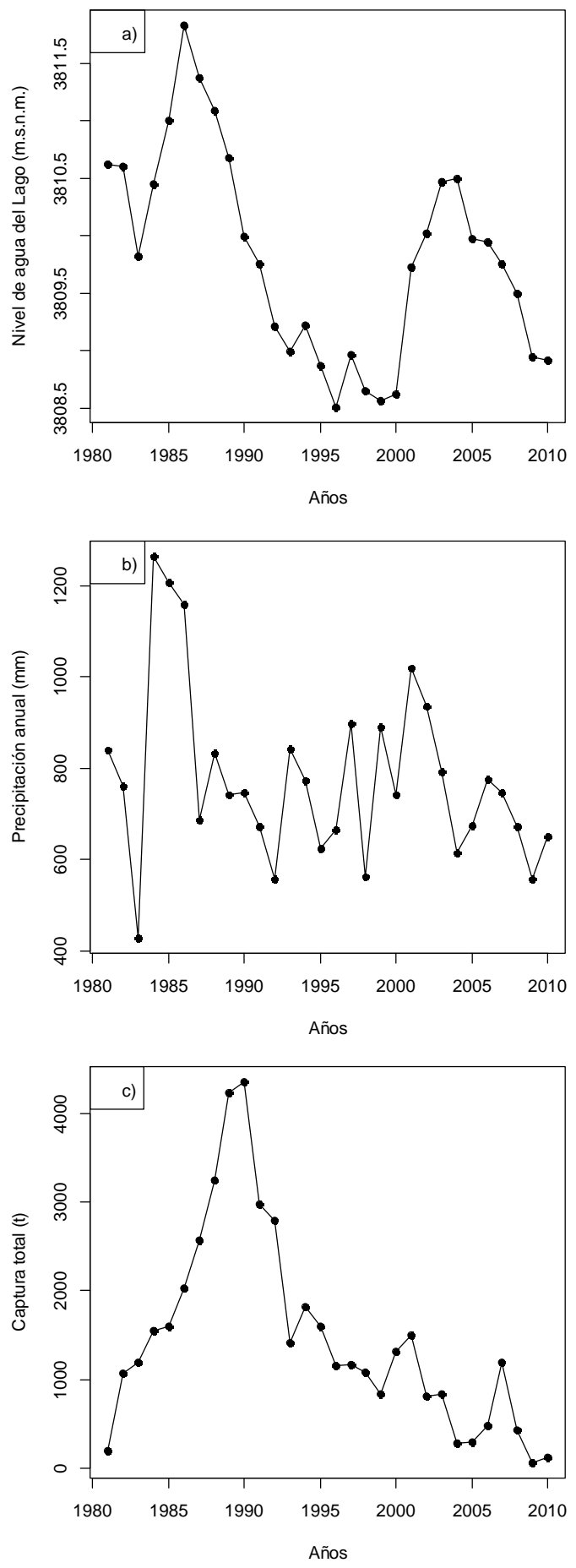

Figura 3. (a) Nivel del Lago Titicaca, (b) Precipitación anual y (c) Desembarque de pejerrey Odontesthes bonariensis (1981 - 2010).

estructura de autocorrelación que el nivel del lago, con $\mathrm{r}=0.73(\mathrm{P}<0.05)$ y $\mathrm{r}=0.59(\mathrm{P}<0.05)$ al primer $\mathrm{y}$ segundo año de desfase. Por último, la serie de tiempo anual de la precipitación no mostró una estructura autocorrelacionada.

Series de tiempo mensuales.

Al observar las series de tiempo mensuales se observa que los cambios en el nivel del lago presentan una variabilidad de mayor frecuencia que los cambios en la precipitación y que ambas series presentan un marcado componente de fluctuación estacional. En la escala mensual, la señal estacional del nivel del lago indica que entre diciembre y abril se presenta un fuerte ascenso de las aguas producto del máximo de aportes globales de las lluvias y de los tributarios (Ríos Ramis, Coata, Ilave y Huancané). Entre mayo a diciembre se presenta el descenso debido a la ausencia de lluvias y a la fuerte evaporación que se produce en el lago (Figura 5a). En el caso de la precipitación, se observa una señal estacional con la mayor precipitación sobre el lago desde diciembre a marzo, que corresponde al verano austral. En tanto, de mayo a junio las precipitaciones decaen prácticamente a cero (Figura 5b).

En el caso de los desembarques de pejerrey, la señal estacional revela altos desembarques durante la época reproductiva (agosto - setiembre) y durante la época de reclutamiento (enero - abril), con los más bajos desembarques en junio (IMARPE, 2009). Se destaca que solo se cuenta con registros mensuales de desembarque de pejerrey desde 1990, observándose una fuerte tendencia a disminuir desde 1990 a 1995 , para posteriormente disminuir de una forma gradual con aumentos de poca importancia en 2001 y 2007. En estos periodos se observaron los residuales más importantes, no explicados por la tendencia ni la señal estacional.

$\underline{\text { Relación de corto plazo entre nivel del lago y la }}$ precipitación con desembarques del pejerrey.

En la escala mensual, los residuales del nivel del lago se correlacionan significativamente con la precipitación hasta por tres meses de retraso, con un máximo a los dos meses (Figura 6a). La correlación cruzada entre los residuales del nivel del lago y de desembarque de pejerrey no evidenció correlaciones importantes (Figura 6b), a excepción del efecto de la precipitación con un retraso de 18 meses (Figura 6c). En la correlación cruzada nivel-desembarques destaca la presencia de correlaciones significativas en retrasos negativos de la variable independiente y que carecen de sentido. En efecto, no es lógico establecer que los desembarques están determinando incrementos en el nivel del lago después de ocho meses.

Procesos de expansión y contracción de la zona litoral del Lago Titicaca

Las fluctuaciones del nivel de agua del Lago Titicaca a nivel de la zona litoral determinan procesos de expansión y/o contracción. De acuerdo con Wirrmann (1991), el descenso de un metro en el nivel ocasiona una disminución de la superficie del agua de $1000 \mathrm{~km}^{2}$ y la disminución del volumen de $8 \mathrm{~km}^{3}$ 
Precipitación-Nivel Lago

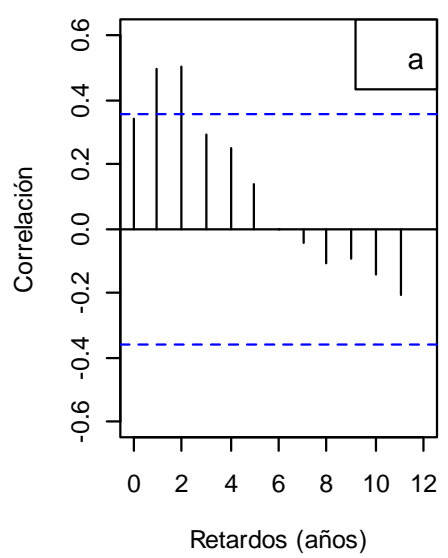

Nivel Lago-Pejerrey

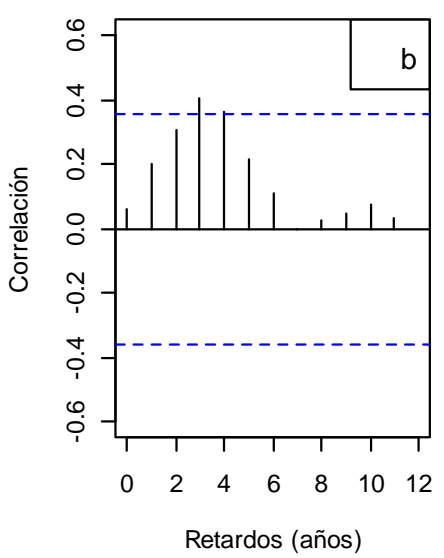

Precipitación-Pejerrey

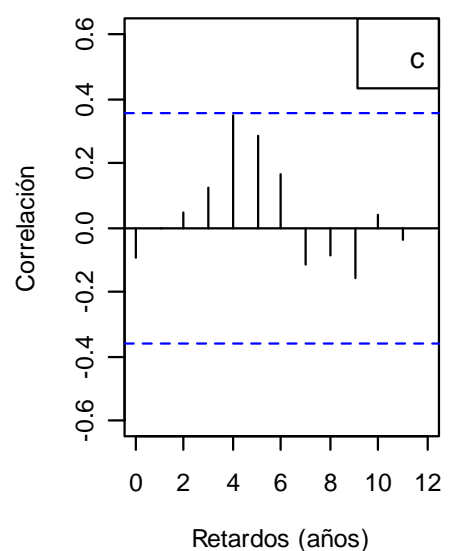

Figura 4. Función de correlación cruzada de los residuales de a) Precipitación Anual - Nivel del lago, b) Nivel del lago - Desembarque pejerrey anual y c) Precipitación Anual - Desembarque pejerrey anual; la línea punteada indica el nivel significativo de correlación $(\alpha=0.05)$.

aproximadamente. De las imágenes satelitales digitalizadas de LANDSAT entre el periodo 1981 y 2009 se determinó una relación directa entre el área de inundación con el nivel de agua del Lago Titicaca $(\mathrm{r}=0.97, \mathrm{n}=10)$. Entre 1981 y 1999 , se observó la máxima área de inundación en 1986, con $633 \mathrm{~km}^{2}$ producto del máximo nivel de agua del lago registrado (3 $811.83 \mathrm{msnm}$ ). Las áreas menores ocurrieron en 1983 y 1996, con 172 y $700 \mathrm{~km}^{2}$ por debajo del nivel normal, respectivamente. En años más reciente (2000 - 2009) se observó que la máxima inundación ocurrió en 2004 con $99 \mathrm{~km}^{2}$ y la más baja en 2009 con 536 $\mathrm{km}^{2}$, por debajo del nivel normal (Figura 7). Se observa que las áreas inundables tienden a ocurrir en el sector peruano del lago, principalmente en la Bahía de Puno, y las zonas Norte y Sur del lago.

\section{Discusión.}

Las fluctuaciones en el nivel de agua de los lagos y la precipitación pueden influir en la productividad de las poblaciones de peces. Se ha determinado que las fluctuaciones del nivel de agua del Lago Titicaca están moduladas principalmente por los regímenes de precipitación que se presentan en la cuenca del lago
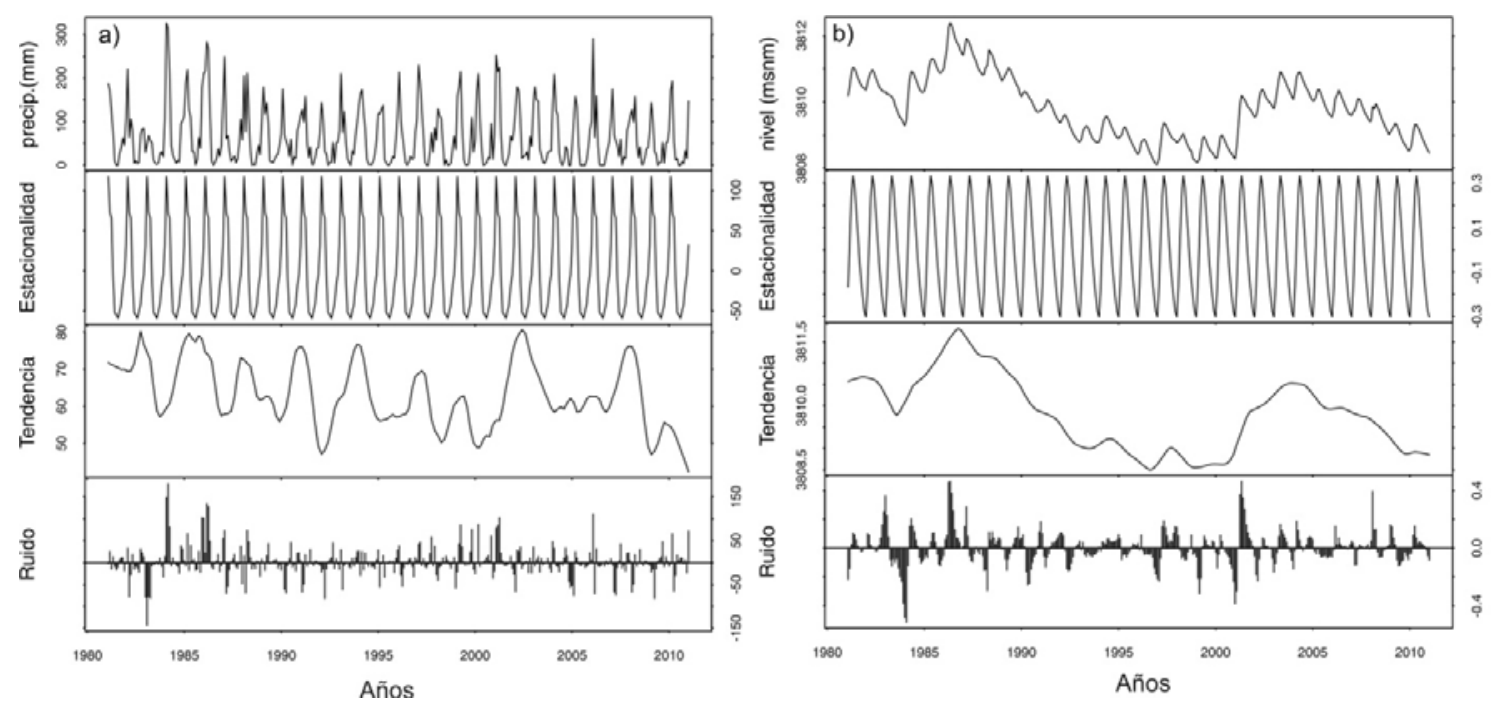

Figura 5. Descomposición de las series de tiempo mensual de las variables ambientales usando un suavisador (STL), entre 1981 - 2010. a) Serie de tiempo del nivel del lago, b) Serie de tiempo de precipitación. 
(Roche et al., 1991; Paredes \& Gonfiantini 1999; Garreaud \& Aceituno, 2001). Las fluctuaciones estacionales del nivel de agua del lago son determinadas por el balance entre los aportes y las pérdidas en agua, siendo en abril, el máximo nivel anual del lago, al final de periodo de lluvias y del aporte de los ríos tributarios, y el mínimo ocurre en diciembre, justo antes de las lluvias del año (Roche et al., 1991). En la escala interanual, la correlación cruzada indica que la precipitación influye significativamente en el nivel de agua del lago hasta después de dos años; mientras que en el corto plazo (meses) influye durante dos a tres meses. La autocorrelación en el nivel de agua del lago corrobora que las fluctuaciones anuales que se presentan en el lago productos de las lluvias que ocurren con una mayor intensidad entre diciembre y febrero (Garreaud \& Aceituno, 2001).

Se observó también que el nivel del lago presenta fluctuaciones de baja frecuencia que podrían estar relacionado con las fluctuaciones El Niño-Oscilación del Sur. Según, Garreaud \& Aceituno (2001) y Garreaud et al. (2003) las lluvias interanuales en el altiplano están relacionadas con el índice de oscilación del sur - El Niño (ENSO), esto es explicado por el calentamiento (enfriamiento) generalizado de la troposfera tropical durante las fases negativas (positivas) del ENSO y la asociación fuerte (débil) sobre el oeste de los Andes central. Entre 1981 y 2010 en el altiplano peruano - boliviano se presentaron dos eventos de El Niño muy intensos en 1982-83 y 19971998, los cuales coinciden con periodos de baja precipitación anual (Maldonado \& Calle, 1998).

No obstante, existen otros factores que modulan las fluctuaciones del nivel de agua del Lago Titicaca, tal como el aporte de los ríos y arroyos en 53\% de agua al lago, los ríos más grandes son el Ramis, Coata, Ilave, Huancané, y Suches que ingresan al lago Grande; y el río Tiwanaco que desemboca en el lago Menor, con descargas máximas al lago entre enero a abril y el mínimo en época seca (junio - julio) (Roche et al., 1991). Asimismo, la remoción de aguas del lago se debe a la evaporación que representa el $91 \%$, que es el responsable del balance del agua y posiblemente el primero que muestra la variabilidad interanual (Roche et al., 1991; Paredes \& Gonfiantini, 1999).

Efecto del nivel del lago y precipitación sobre la disponibilidad del pejerrey.

Con el fin de estimar los desembarques del pejerrey, se estimaron dos modelos de regresión lineal simple con los desembarques anuales de pejerrey en función del nivel de agua del lago, desfasados en tres años, para el periodo 1981-2004 y para el periodo 2005-2010. Se encontró que la relación fue más significativa en el primer periodo que en el segundo. Es probable que el cambio en la relación sea consecuencia de la importante disminución de la abundancia o productividad de esta especie, ya que los desembarques de pejerrey comenzaron a ser más bajos de lo esperado en el segundo periodo. Aparentemente, la leve recuperación de los desembarques de pejerrey en 2007 y 2008 se debe al desembarque de la zona Norte (Huancané) principalmente $(40.2 \%)$, lo que demuestra la virtual ausencia de pejerrey en otros sectores.

En los residuales mensuales no se encontró una asociación significativa entre el nivel de agua de lago y los desembarques. Al parecer, la ampliación de la zona litoral por procesos de crecida del nivel de agua del lago estaría más bien favoreciendo el éxito del reclutamiento del pejerrey principalmente por dos mecanismos: (i) por la disponibilidad de alimento
Precipitación-Nivel Lago

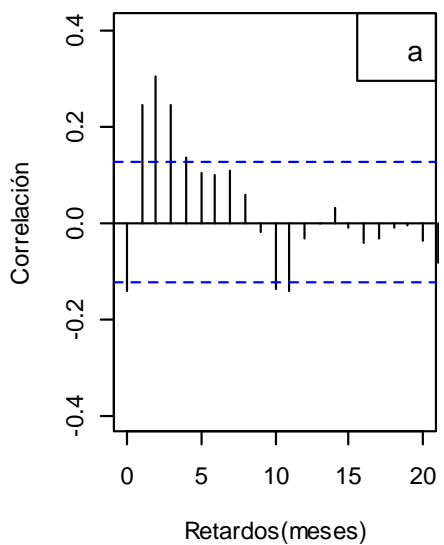

Nivel Lago-Pejerrey

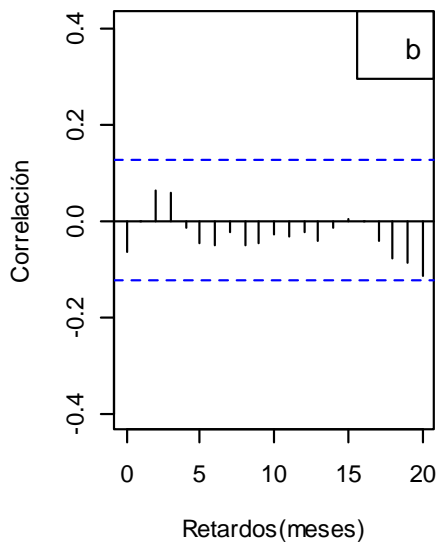

Precipitación-Pejerrey

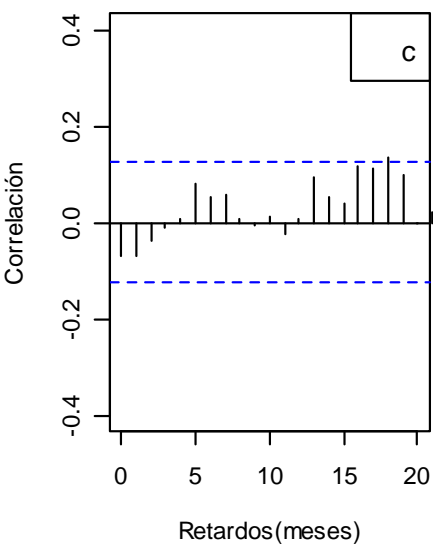

Figura 6. Función de correlación cruzada de los residuales de a) Precipitación mensual - Nivel del lago, b) Nivel lago - Desembarque pejerrey mensual y c) Precipitación mensual - desembarque pejerrey mensual, la línea punteada indica el nivel significativo de correlación con $\alpha=0.05$. 
natural (fitoplancton y zooplancton) y (ii) mediante el refugio frente a depredadores.

La crecida del nivel de agua del lago genera ampliación de las áreas inundables, principalmente en las zonas de la desembocadura del río Ramis, del Coata (Bahía de Puno) y del Ilave, debido a que la topografía de la zona litoral es extensa y plana (Figura 7). Leviel \& Orlove (1990) indican que cuando disminuye el nivel de agua del lago, los campesinos que habitan en la zona litoral tienden a aprovechan esta zona para realizar cultivo de tubérculos y hortalizas, para lo cual utilizan productos fertilizantes naturales que aportan de materia orgánica (estiércol), convirtiéndose en áreas ricas en nutrientes; cuando el lago recupera su nivel, el cual es aprovechado por el fitoplancton y el zooplancton, que es alimento para los alevines y juveniles del pejerrey. Asimismo, la zona litoral actúa como una zona de refugio para los pequeños peces (alevines y juveniles) contra los peces grandes (pejerrey adulto, trucha y Orestias spp). Según Grossman (2001) indica que el pejerrey de tallas mayores a $35 \mathrm{~cm}$ LS son carnívoros con tendencia al canibalismo sobre los peces pequeños de la misma especie.

Por último, por la condiciones ambientales favorables, principalmente la temperatura del agua que en la época de reclutamiento (noviembre - marzo) fluctúa entre 16 y $20{ }^{\circ} \mathrm{C}$ (IMARPE, 2009) con presencia de grandes cantidades de alevinos y juveniles en la zona litoral. IMARPE (2010) de la a)

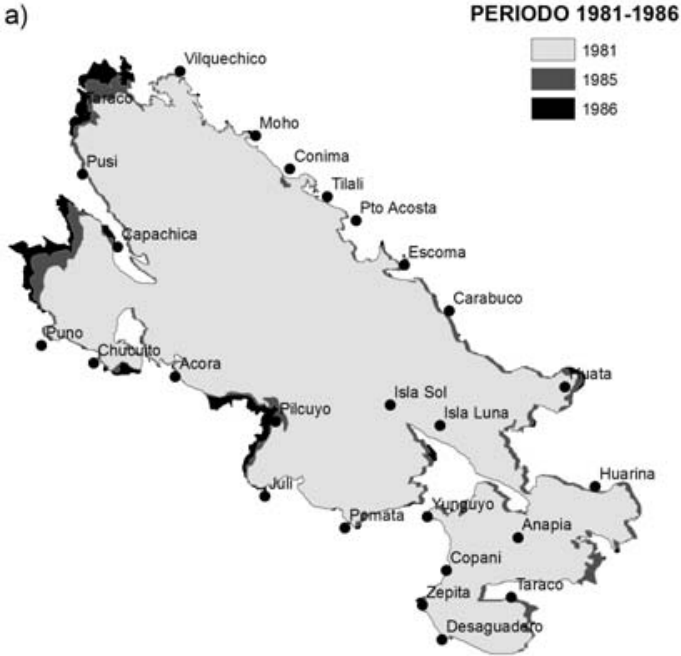

c)

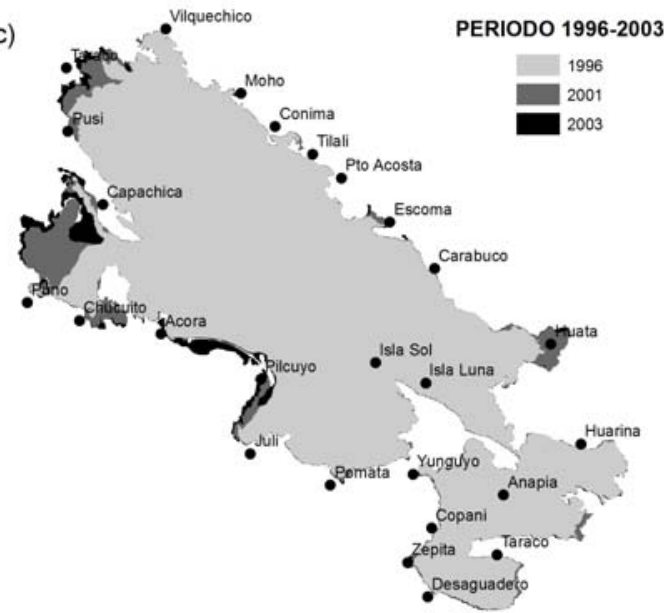

b)

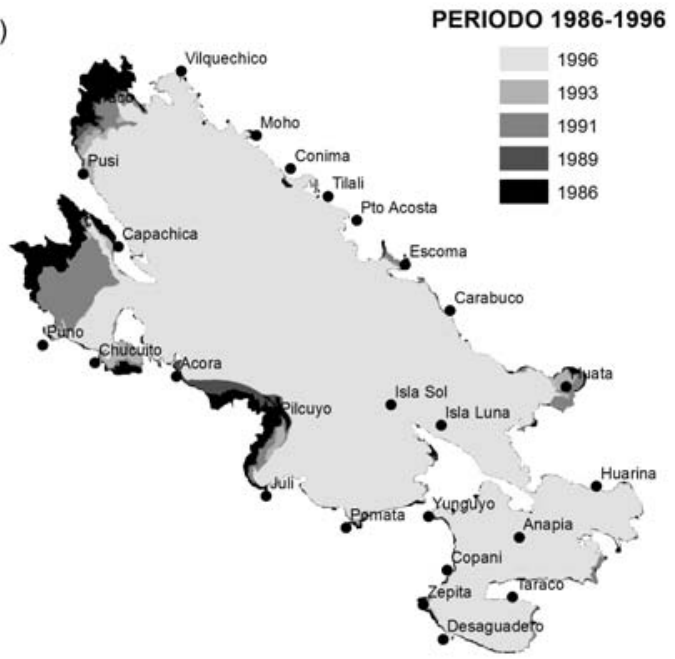

d)

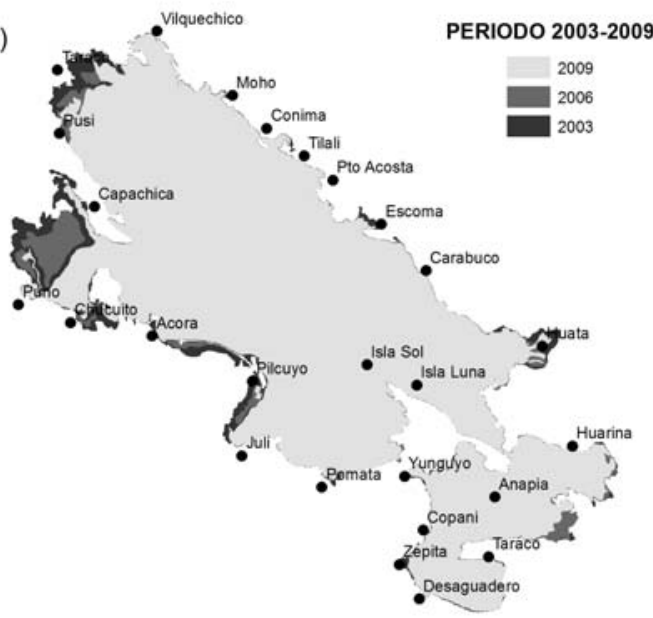

Figura 7. Áreas inundables en el Lago Titicaca productos de las fluctuaciones interanuales del nivel de agua del lago a) periodo 1981-1986 b) periodo $1986-1996$ c) periodo $1996-2003$ y d) periodo $2003-2009$. 
pesca experimental con chinchorro de playa en zona litorales $(<5 \mathrm{~m})$ en diciembre del 2009 determinó presencia de pejerreyes con una amplitud de tallas de 2.5 a $12.5 \mathrm{~cm}$ de LT y una distribución bimodal en 4.5 $\mathrm{cm}$ y $9.5 \mathrm{~cm}$ de LT.

El Instituto del Mar del Perú, a través de la evaluación hidroacústica en 2010, determinó una biomasa de pejerrey en $8491 \mathrm{t}$; lo que representa un decremento de $57.5 \%$ respecto de lo registrado en 1985. De forma similar los desembarques anuales entre 1981 y 2010 muestran una tendencia a la disminución, debido principalmente a las fluctuaciones del nivel de agua del Lago Titicaca y una sobrepesca a nivel de los juveniles. Además, se adiciona la falta de un ordenamiento pesquero binacional entre Perú y Bolivia; y debilidad en el sector peruano en el control y cumplimiento de las normas legales vigentes por los pescadores artesanales sobre tallas mínimas de captura $(>22.5 \mathrm{~cm} \mathrm{LT})$, tamaños de abertura de mallas $(42.3 \mathrm{~mm}$ de luz de malla cortinera, Resolución Ministerial N ${ }^{\circ}$ 271-2010PRODUCE), vedas por reproducción y reclutamiento. Esta situación podría haber interrumpido los ciclos naturales de buenos desembarques del pejerrey asociados al nivel de agua del lago.

\section{Conclusiones.}

Los cambios en el nivel del lago están asociados significativamente con la precipitación hasta después de dos años.

Entre 1981 y 2004 se observó una correlación directa entre el nivel del lago y las capturas de pejerrey, mientras que entre 2005 y 2010 la relación se deterioró, posiblemente debido a la sobrepesca, contaminación u otros factores no considerados.

A escala anual, el análisis de correlación cruzada determinó que las capturas de pejerrey aumentarían tres años después de eventos de crecida del nivel del lago. Esta asociación podría deberse a la disponibilidad de alimento, refugio frente a depredadores o condiciones ambientales favorables.

\section{Agradecimientos.}

El autor principal agradece a los docentes del Programa de Magíster en Ciencias con Mención en Pesquerías de la Universidad de Concepción (Chile) por su apoyo en la realización del presente trabajo. Asimismo, a los colegas del Instituto del Mar del Perú - Laboratorio Continental de Puno y a la Dirección Regional de la Producción Puno. Finalmente, agradecemos los comentarios y sugerencias de los revisores anónimos de este artículo.

\section{Literatura citada.}

Alheit J. \& Bakun A. 2010. Population synchronies within and between ocean basins: apparent teleconnections and implications as to physical-biological linkage mechanisms. Journal of Marine Systems. 79:267-285.
Brockwell P. \& Davies R. 2002. Introduction to times series and forecasting. Segunda Edición. Springer. New York. $434 \mathrm{pp}$.

Calsina C., Salas R., Limachi J. \& Tambo E. 2001. Evaluación del potencial de especies introducidas en el ámbito Boliviano del sistema TDPS. Proyecto PER/98/G-32 Conservación de la biodiversidad en la cuenca del lago Titicaca. Subcontrato 24.25. Informe Final. 55 pp.

Cleveland R., Cleveland W., Mcrae J. \& Terpenning I. 1990. STL: a seasonal-trend decomposition procedure based on Loess. Journal of Official Statistics. 6:3-73.

Deguen M., Huerta R., Apaza F., Vilcherrez J., Galves J. \& Martinez V. 2002. Evaluación del potencial de promoción de pesca de especies introducidas. Proyecto PER/98/G-32 Conservación de la biodiversidad en la cuenca del lago Titicaca. Subcontrato 24.25. Informe Final. $168 \mathrm{pp}$.

Flores S. 2010. Estimación de edad y crecimiento del pejerrey Odontesthes bonariensis, mediante la fórmula de Von Bertalanffy en cuatro zonas del Lago Titicaca. Tesis, Universidad Nacional del Altiplano, Puno. 120 $\mathrm{pp}$.

Fréon P., Bouchon M., Mullon C., García C. \& Niquen M. 2008. Interdecadal variability of anchoveta abundance and overcapacity of the fishery in Peru. Progress in Oceanography. 79:401-412.

Garraeaud R., Vuille M. \& Clements A. 2003. The climate of the Altiplano: Observed current conditions and past change mechanisms. Palaeogeography, Palaeoclimatology, Palaeoecology. 3054:1-18.

Garreaud R. \& Aceituno P. 2001. Interanual Rainfall Variability over the South American Altiplano. Journal of Climate. 14:2279-2789.

Grossman F. 2001. Fundamentos Biológicos, Económicos y Sociales para una Correcta Gestión del Recurso Pejerrey. Editorial Astyanax. Buenos Aires, Argentina. $246 \mathrm{pp}$.

Hahmenberger M. 2003. Summertime precipitation variability and atmospheric circulation over the south American Altiplano: Effects of lake Titicaca and salar de Uyuni. Oklahoma Weather Center Research Experiences for Undergraduates Program, Summer. $10 \mathrm{pp}$.

Instituto Del Mar Del Perú. 2008. Anuario Científico Tecnológico IMARPE. Enero - Diciembre. Volumen 8. ISSN 1813-2103. $176 \mathrm{pp}$.

Instituto Del Mar Del Perú. 2009. Anuario Científico Tecnológico IMARPE. Enero - Diciembre. Volumen 9. ISSN 1813-2103. $182 \mathrm{pp}$.

Instituto Del Mar Del Perú. 2010. Anuario Científico Tecnológico IMARPE. Enero - Diciembre. Volumen 10. ISSN 1813-213. 195 pp.

Leira M. \& Cantonati M. 2008. Effects of water-level fluctuations on lake: an annotated bibliography. Hydrobiologia 613:171-184.

Leviel D. \& Orlove B. 1990. Local control of aquatic resources: Community and Ecology in Lake Titicaca, Peru. American Anthropologist. 92:362-382.

Loubens G. \& Osorio F. 1991. Especies introducidas: Basilichthyes bonariesis. En: El Lago Titicaca: Síntesis del Conocimiento Limnológico Actual. Dejoux \& A. Iltis (eds).OSTOM-HISBOL, La Paz, Bolivia. 584(Cap. VI.5b): 431-449. 
Maldonado R. \& Calle S. 1998. Comportamiento de las precipitaciones en el sector del Lago Titicaca (Bolivia) durante "El Fenómeno El Niño". Bulletin de l'Institut Français d'Etudes Andines. 27:699-707.

Ministerio de la Producción, Gobierno Regional de Puno y Agencia Española de Cooperación Internacional para el Desarrollo. 2009. "informe final convenio 2007: informe de actividades convenio IMARPE-FONCHIP 20082009". Proyecto-PROPESCA. 37 pág.

Paredes M. \& Gonfiantini G. 1999. Lake Titicaca: historian and current studies. Water and Environment News. International Atomic Energy Agency. Quarterly No 8/9, December 1999. 6-8 pp.

Pyper J. \& Peterman R. 1998. Comparison of methods to account for autocorrelation in correlation analysis of fish data. Canadian Journal of Fisheries and Aquatic Sciences. 55:2127-2140.

Quiñones R. \& Montes R. 2001. Relationship between freshwater input to the coastal zone and historical landings of the benthic/demersal fish Eleginops maclovinus in central-south Chile. Fisheries Oceanography. 10:311-328.
R Development Core Team. 2010. R: A language and environment for statistical computing. R Foundation for Statistical Computing, Vienna, Austria. URL http://www.R-project.org.

Roche M., Bourges J., Cortes J. \& Mattos R. 1991. Climatología e Hidrología de la Cuenca de lago Titicaca. In El Lago Titicaca: Síntesis del Conocimiento Limnológico Actual. Dejoux \& A. Iltis (eds).OSTOMHISBOL, La Paz, Bolivia. 584(Cap. IV.1): 83-104.

Vaux P., Wurtsbaugh W., Trevino H., Mariño L., Bustamante E., Torres J., Richerson P. \& Alfaro R. 1988. Ecology if the Pelagic Fishes of Lake Titicaca, Perú -Bolivia. Biotopa. 20:220-229.

Wirrmann D. 1991. Capítulo II Morfología y Batimetría. In El Lago Titicaca: Síntesis del conocimiento limnológico actual. Dejoux \& A. Iltis (Eds).OSTOM-HISBOL, La Paz, Bolivia. Pág. 584:31-38.

Yucra A. 1993. Reproducción Artificial del Odontesthes bonariensis pejerrey. Tesis de Licenciatura. Universidad Nacional del Altiplano, Facultad de Ciencias Biológicas. $45 \mathrm{pp}$.

\footnotetext{
${ }^{1}$ Programa de Magíster en Ciencias con Mención en Pesquerías, Universidad de Concepción, Concepción, Chile. Autor correspondiente: rchurac@hotmail.com.

${ }^{2}$ Departamento de Oceanografía, Facultad de Ciencias Naturales y Oceanográficas, Universidad de Concepción, Concepción, Chile.

${ }^{3}$ Centro de Investigaciones en Modelado Oceanográfico y Biológico Pesquero, Instituto del Mar del Perú IMARPE.

${ }^{4}$ Laboratorio Continental de Puno, Instituto del Mar del Perú - IMARPE.
} 